\title{
Pulmonary Tumor Thrombotic Microangiopathy Caused by Recurrent Gastric Cancer 26 Years After Total Gastrectomy
}

\author{
Tadashi Yuguchi ${ }^{1}$, Hiroyuki Sano ${ }^{1}$, Kenji Nakajima $^{1}$ and Yoshihiro Ikura ${ }^{2}$
}

\begin{abstract}
:
Pulmonary tumor thrombotic microangiopathy (PTTM) is a rare cancer-related complication that induces pulmonary hypertension (PH). PTTM can be caused by recurrent cancer, with 12 years being the longest reported interval from primary cancer to the development of PTTM. We herein report a 74-year-old woman who presented with dyspnea due to PH. The postmortem diagnosis was PTTM caused by recurrent gastric cancer 26 years after total gastrectomy. An autopsy revealed PTTM-specific histological characteristics. Our findings indicate that PTTM should be considered as a diagnosis for patients with a history of cancer who develop PH, even several decades after treatment.
\end{abstract}

Key words: Pulmonary tumor thrombotic microangiopathy, Recurrent gastric cancer, Tumor dormancy

(Intern Med Advance Publication)

(DOI: 10.2169/internalmedicine.8559-21)

\section{Introduction}

Pulmonary tumor thrombotic microangiopathy (PTTM) is a rare and fatal cancer-related pulmonary complication first reported by Von Herbay et al. (1) It is histologically characterized by tumor embolisms and reactive fibrocellular intimal proliferation in small pulmonary arteries and arterioles $(1,2)$. The major histopathological cancer type associated with PTTM is adenocarcinoma, and the primary cancer most frequently originates from the stomach (3).

For the antemortem diagnosis of PTTM, the medical information of patients with active cancer or a history of cancer is crucial for providing an appropriate diagnosis. PTTM can reportedly be caused by recurrent cancers; a recent case reported PTTM associated with recurrent cancer at 12 years after the diagnosis of primary breast cancer (4).

We herein report a case of PTTM caused by ultra-late recurrent gastric cancer treated 26 years previously.

\section{Case Report}

A 74-year-old Japanese woman presented with exertional dyspnea and mild leg edema lasting for 1 month. She had a history of gastric signet ring cell carcinoma, pathologically classified as T1N1M0, 26 years earlier and had been treated with total gastrectomy and lymph node dissection followed by adjuvant chemotherapy. Otherwise, the patient had no remarkable medical problems. She had never smoked. Her blood pressure was normal $(116 / 79 \mathrm{mmHg})$, heart rate was slightly high (115 beats per minute [bpm]), respiratory rate was high (20 times per minute), oxygen saturation was low (peripheral capillary oxygen saturation $\left(\mathrm{SpO}_{2}\right) 83 \%$ on room air), and body temperature was normal $\left(36.4{ }^{\circ} \mathrm{C}\right)$. She had bilateral fine crackles in the lungs, but chest auscultation revealed no abnormal heart sounds or murmurs. We observed distention of the jugular vein and mild pitting edema in both lower legs.

Chest radiography showed bilateral diffuse opacity, which was especially noticeable in the lower lung field with pleural effusion (Fig. 1). An electrocardiogram showed an inverted $\mathrm{T}$ wave in the V1-4 leads, implying right heart overload (Fig. 2). Transthoracic echocardiography revealed an estimated systolic pulmonary artery pressure of $99 \mathrm{mmHg}$ based on the tricuspid regurgitation velocity, which indicated severe pulmonary hypertension $(\mathrm{PH})$. A dilated right ventricle and compressed left ventricle and pericardial effusion were observed. The right ventricular function was found to

${ }^{1}$ Department of Internal Medicine, Division of Cardiovascular Medicine, Aijinkai Takatsuki General Hospital, Japan and ${ }^{2}$ Department of Pathology, Aijinkai Takatsuki General Hospital, Japan

Received: August 24, 2021; Accepted: September 13, 2021; Advance Publication by J-STAGE: October 26, 2021

Correspondence to Dr. Tadashi Yuguchi, yuguchi.tadashi@aijinkai-group.com 


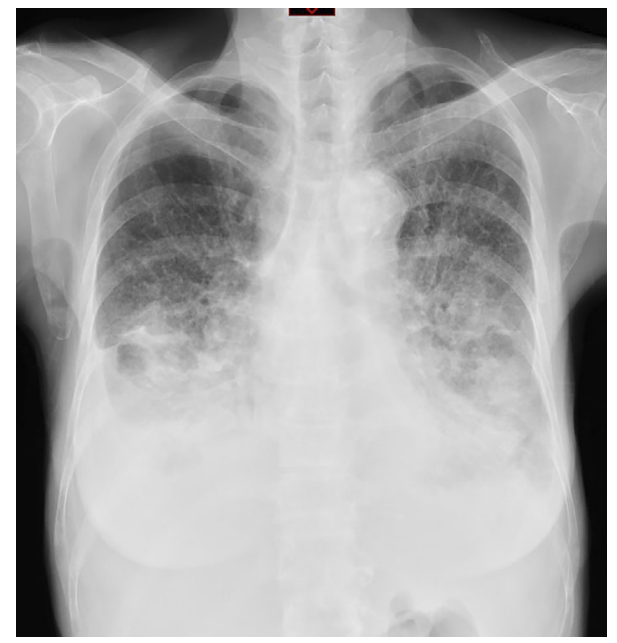

Figure 1. Chest X-ray on admission showed bilateral diffuse opacity, with particularly remarkable findings in the lower lung field with plural effusion.

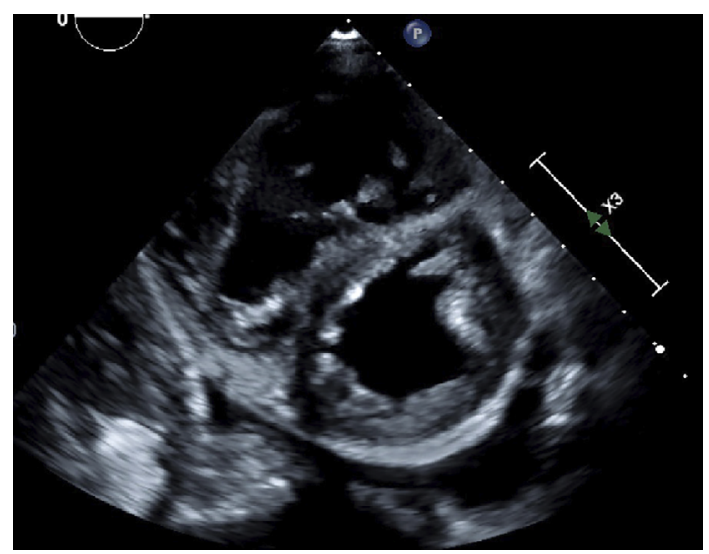

Figure 3. A short-axis view of the transthoracic echocardiogram obtained on admission showed the dilated right ventricle compressing the left ventricle, suggesting $\mathbf{P H}$.

be deteriorated with $13 \mathrm{~mm}$ of tricuspid annular plane systolic excursion, whereas the left ventricular function was normal (Fig. 3). Computed tomography (CT) revealed bilateral diffuse ground-glass opacity and prominent intralobular septal thickening with pleural effusion (Fig. 4A). A contrastenhanced image showed that only one small peripheral pulmonary artery was embolized (Fig. 4B). Blood work showed high D-dimer $(18 \mu \mathrm{g} / \mathrm{mL})$, high brain natriuretic peptide (328.6 pg/mL), and slightly low hemoglobin (11.6 g/dL) levels. However, the kidney, liver, and electrolyte functions were normal. Immunological values, including antineutrophil cytoplasmic antibodies, were normal, as were several tumor biomarkers, including as carcinoembryonic antigen (CEA) and CA19-9. A blood gas analysis at $2 \mathrm{~L} / \mathrm{min}$ $\mathrm{O}_{2}$ showed hypoxemia $\left(\mathrm{PaO}_{2} 65.1 \mathrm{mmHg}\right)$ without hypercapnia $\left(\mathrm{PaCO}_{2} 40.1 \mathrm{mmHg}\right)$.

Thoracentesis of the right pleural effusion was performed on the second hospital day. The drained effusion appeared yellowish, transparent, and serous. A right heart catheterization test conducted on the same day showed a mean pulmo-

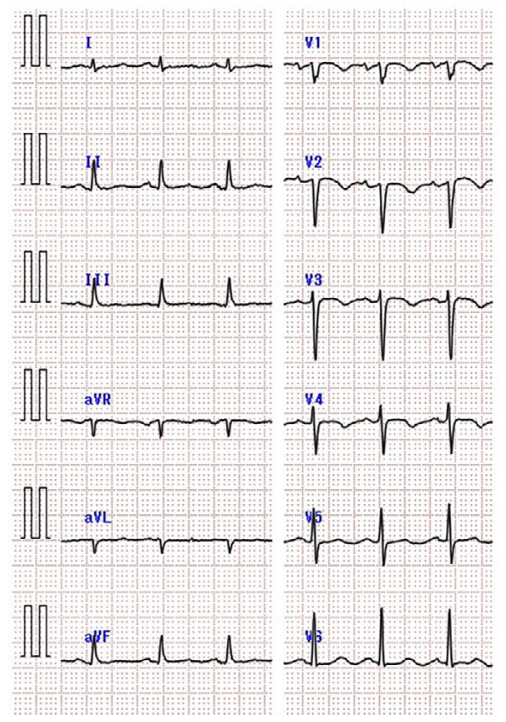

Figure 2. An electrocardiogram on admission showed an inverted $T$ wave in V1-4 leads, suggesting right heart overload.

nary capillary wedge pressure of $9 \mathrm{mmHg}$, mean pulmonary artery pressure of $27 \mathrm{mmHg}$, pulmonary vascular resistance of 483 dynes $\cdot \mathrm{s} \cdot \mathrm{cm}^{-5}$, and cardiac output index of $2.08 \mathrm{~L} / \mathrm{min} /$ $\mathrm{m}^{2}$. These results indicated a mild increase in pulmonary arterial pressure and pulmonary vascular resistance without left ventricular failure. The coronary arteries were intact. Lung ventilation/perfusion scans were not available at our facility and were therefore not performed. Based on the abovementioned history and test results, we made a tentative diagnosis of $\mathrm{PH}$ with a non-identified cause, but likely differential diagnoses included PTTM, chronic thromboembolic pulmonary hypertension, pulmonary veno-occlusive disease, and connective tissue disease-related $\mathrm{PH}$

We applied conservative treatment for $\mathrm{PH}$, including diuresis for volume overload, administration of an inotrope agent for right heart dysfunction, oxygen for hypoxemia, and unfractured heparin for thromboembolization. The combination of these treatments gradually attenuated $\mathrm{PH}$, but the patient's symptoms and general condition showed no improvement. On the seventh hospital day, a cytological examination revealed malignant pleural effusion with CEApositive adenocarcinoma. This result confirmed that PTTM was the main cause of the disease. Contrast-enhanced CT of the chest and abdomen could not determine the primary cancer. The patient was too ill to undergo further inspections. Pulmonary vasodilators, including the endothelin receptor antagonist bozentan and the phosphodiesterase-5 inhibitor tadalafil, were added to the conservative treatment. Although the tyrosine kinase inhibitor imatinib has been reported to be effective, it was not used because our hospital ethics committee determined that her poor performance status made her ineligible for its initiation.

The patient continued the described treatment plus palliative care but ultimately died on the 30th hospital day. An autopsy indicated that PTTM was caused by signet ring cell carcinoma. Although the stomach had been completely re- 


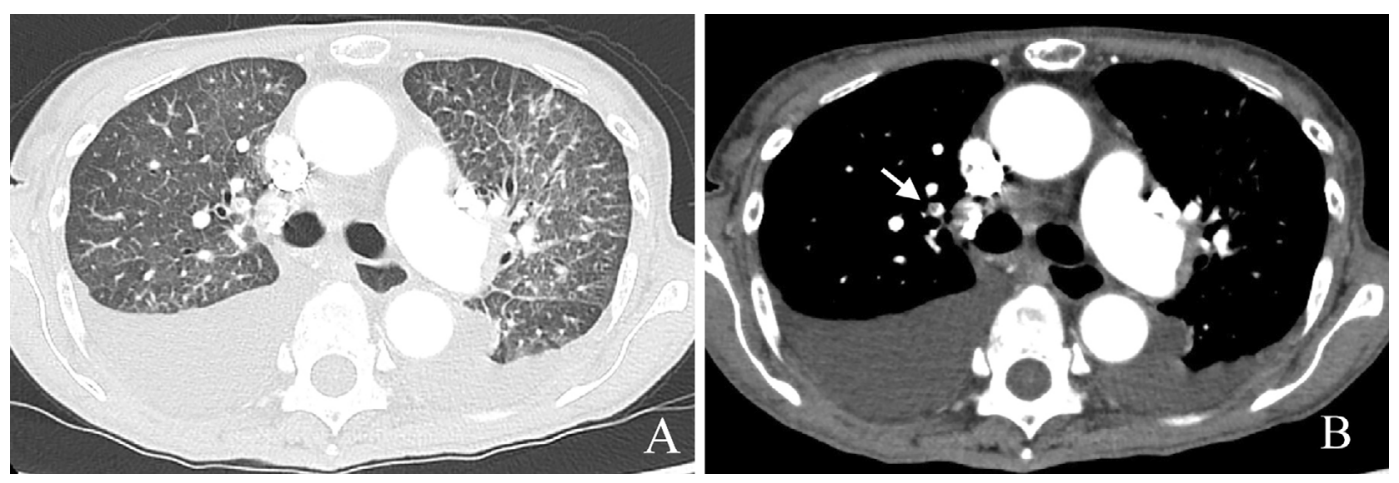

Figure 4. Computed tomography on admission. (A) The lung window image shows bilateral diffuse ground-glass opacity and intralobular septal thickening with plural effusion. (B) The mediastinal window image shows only one small embolized pulmonary artery (white arrow).
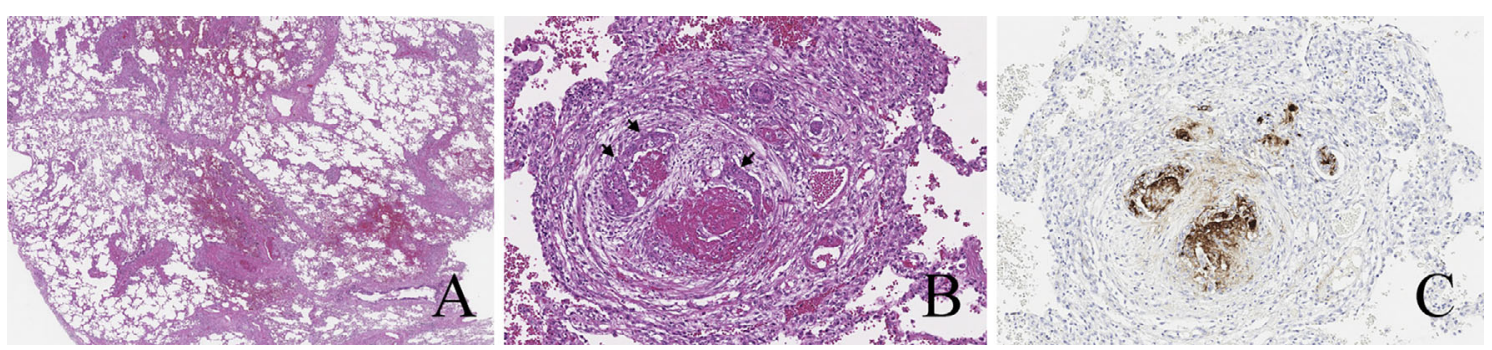

Figure 5. Histologic findings of the autopsied lung. (A) Patchy alveolar hemorrhaging is visible in a low-magnification view. Hematoxylin and Eosin (H\&E) staining; original magnification 4×. (B) Fibro-cellular intimal proliferation and thrombosis associated with tumor embolism (arrows) were observed in the center of the hemorrhagic lesion. H\&E staining; original magnification 40×. (C) Immunohistochemically, embolic tumor cells were positive for the gastric epithelial marker MUC5AC. Immunoperoxidase stain; original magnification $40 \times$.

moved, and no obvious recurrence was observed in either the anastomosis site or gastrointestinal tract, we considered the tumor cells to be derived from the previous gastric cancer. In addition to PTTM, metastasis of signet ring cell carcinoma was confirmed in the spleen, pleura, para-aortic lymph nodes, and bone marrow. A low-magnification histological view of the lungs showed many foci of patchy alveolar hemorrhaging (Fig. 5A). In the center of each hemorrhagic lesion, we observed small arteries/arterioles occluded by tumor emboli that comprised signet ring cell carcinoma, which was positive for the gastric epithelial marker MUC5 AC (Fig. 5B \& C). In addition to tumor embolism, microhemorrhaging, thrombosis, and reactive intimal proliferation have been deemed unique to PTTM and reportedly contribute to vascular occlusion (Fig. 5B). Additional immunohistochemical markers, such as PD-L1 and HER2, were evaluated, but no definitive cause of tumor dormancy or reproliferation was established.

\section{Discussion}

PTTM is a rare disease that causes cancer-related PH and is known for its poor prognosis. A recent systematic review reported that the average patient age was 56 years old, and
$56 \%$ were men; gastric adenocarcinoma was diagnosed as primary cancer in $59 \%$ of cases. The average length of time from the primary malignancy to presenting with PTTM is 3.5 (range: 0.1-12) years (3). The antemortem diagnosis of PTTM is difficult. PTTM is histologically characterized by tumor embolisms and reactive fibrocellular intimal proliferation in small pulmonary arteries and arterioles $(1,2)$. Lymphangitic carcinomatosis does not usually cause significant PH in isolation but often coexists with PTTM. Pulmonary tumor embolization, a similar disease, is characterized by the occlusion of the small pulmonary arteries by cohesive tumor cells and can only be distinguished from PTTM by a histological examination (5).

It is important that PTTM be diagnosed at an early stage because the median survival time is only three weeks from the onset of symptoms. As in this case, patients with PTTM mostly present with cough (85\%), dyspnea (94\%), and hypoxemia (95\%), which are not specific for PTTM. However, the chest CT findings observed in this case, such as groundgrass opacity and septal thickening, help with the diagnosis of PTTM specifically (3). An early antemortem diagnosis of PTTM can facilitate primary cancer-specific therapies, including surgery, chemotherapy, and radiation. To our knowledge, only 22 antemortem PTTM cases have been diagnosed 
pathologically or clinically (3). The medical information of patients with active cancer or a history of cancer is particularly important for providing an accurate diagnosis for PTTM, as it may also be caused by recurrent cancers. In the current case, although the patient had a medical history of gastric cancer, we did not suspect a diagnosis of PTTM caused by recurrent gastric cancer. Although previous reports have documented PTTM cases associated with recurrent cancer, the longest reported interval from primary cancer to development of PTTM is 12 years (6).

Gastric cancer recurrence usually appears within the first two years post-gastrectomy. Late recurrence is uncommon, with only $10.8 \%$ of cases recurring after 5 years and fewer than $1 \%$ after 10 years (7). To our knowledge, there are only 3 other cases of gastric cancers recurring after 20 years (8-10). Despite the results of our thorough immunohistochemical examination, tumor dormancy can explain the ultra-late recurrence of cancer that we observed in this case. Tumor dormancy with hidden disseminated tumor cells may occur without symptoms and can be untraceable over a prolonged period. Cancer cells can become dormant at the onset of disease or after the initial therapeutic treatment and remain dormant for years or even decades after treatment (11). Metastasis to the bone marrow seems to be particularly related to tumor dormancy. Many factors in the bone microenvironment, including angiogenesis, immune surveillance, and hypoxia, are thought to regulate tumor cell entry and exit from dormancy in different bone marrow niches (12). The present case also demonstrated bone marrow metastasis in addition to PTTM, suggesting that tumor dormancy might have been involved in recurrence. We speculate that gastric adenocarcinoma first metastasized to the bone marrow at an early stage and then remained dormant for more than two decades. After 26 years, the cancer reactivated and recurred as PTTM.

The lack of medical information concerning the gastric cancer that had occurred 26 years earlier limited our ability to clarify the relationship between the primary and metastasis cancer or elucidate the mechanism of tumor dormancy. Further studies, including gene mutation examinations, are needed to better understand the mechanism of PTTM caused by ultra-late recurrent cancer and its relationship with tumor dormancy.

In summary, we report a case of PTTM caused by ultralate recurrent gastric cancer 26 years after its primary onset. We suspected an antemortem diagnosis of PTTM but were unable to identify the primary cancer. Our findings show that even cancers that were treated more than two decades ago can underlie the development of PTTM. The earlier identification of the primary cancer and diagnosis would have encouraged us to introduce more specific therapy, including chemotherapy, corticosteroids, and imatinib.

The authors state that they have no Conflict of Interest (COI).

\section{Acknowledgements}

We would like to express my gratitude to my supervisor, Hideyuki Takaoka. We would also thank Editage (www.editage.c om) for the English language editing.

\section{References}

1. von Herbay A, Illes A, Waldherr R, Otto HF. Pulmonary tumor thrombotic microangiopathy with pulmonary hypertension. Cancer 66: 587-592, 1990.

2. Sato N, Tasaki T, Noguchi H, et al. The pathological challenge of establishing a precise diagnosis for pulmonary tumour thrombotic microangiopathy: identification of new diagnostic criteria. Histopathology 74: 892-901, 2019.

3. Godbole RH, Saggar R, Kamangar N. Pulmonary tumor thrombotic microangiopathy: a systematic review. Pulm Circ 9: I-13, 2019.

4. Fukada I, Araki K, Kobayashi K, et al. Imatinib could be a new strategy for pulmonary hypertension caused by pulmonary tumor thrombotic microangiopathy in metastatic breast cancer. Springerplus 5: 1582, 2016.

5. Chinen K, Tokuda Y, Fujiwara M, Fujioka Y. Pulmonary tumor thrombotic microangiopathy in patients with gastric carcinoma: an analysis of 6 autopsy cases and review of the literature. Pathol Res Pract 206: 682-689, 2010.

6. Price LC, Wells AU, Wort SJ. Pulmonary tumor thrombotic microangiopathy. Curr Opin Pulm Med 22: 421-428, 2016.

7. Moon YW, Jeung HC, Rha SY, et al. Changing patterns of prognosticators during 15-year follow-up of advanced gastric cancer after radical gastrectomy and adjuvant chemotherapy: a 15-year follow-up study at a single korean institute. Ann Surg Oncol 14: 2730-2737, 2007.

8. Blanchette P, Lipton JH, Barth D, Mackay H. Case report of very late gastric cancer recurrence. Curr Oncol 20: 161-164, 2013.

9. Okugawa Y, Toiyama Y, Inoue Y, et al. Late-onset peritoneal recurrence of advanced gastric cancer 20 years after primary resection. World J Surg Oncol 24: 104, 2010.

10. Kawabe T, Yoshinaga M, Kitamura Y, et al. Recurrent advanced gastric cancer diagnosed 20 years after partial gastrectomy. Nihon Shokakibyo Gakkai Zasshi 108: 238-244, 2011.

11. Wang SH, Lin SY. Tumor dormancy: potential therapeutic target in tumor recurrence and metastasis prevention. Exp Hematol Oncol 2: 29, 2013.

12. Mayhew V, Omokehinde T, Johnson RW. Tumor dormancy in bone. Cancer Rep (Hoboken) 3: e1156, 2020.

The Internal Medicine is an Open Access journal distributed under the Creative Commons Attribution-NonCommercial-NoDerivatives 4.0 International License. To view the details of this license, please visit (https://creativecommons.org/licenses/ by-nc-nd/4.0/).

\section{(C) The Japanese Society of Internal Medicine} Intern Med Advance Publication 